\title{
Isotope effects on the lattice parameter of cubic $\mathrm{SiC}$
}

\author{
Carlos P. Herrero and Rafael Ramírez \\ Instituto de Ciencia de Materiales de Madrid, Consejo Superior de Investigaciones \\ Cientificas (CSIC), Campus de Cantoblanco, 28049 Madrid, Spain \\ Manuel Cardona \\ Max-Planck-Institut für Festkörperforschung, Heisenbergstrasse 1, 70569 Stuttgart, Germany
}

(Dated: September 9, 2018)

\begin{abstract}
Path-integral molecular dynamics simulations in the isothermal-isobaric (NPT) ensemble have been carried out to study the dependence of the lattice parameter of $3 \mathrm{C}-\mathrm{SiC}$ upon isotope mass. This computational method allows a quantitative and nonperturbative study of such anharmonic effect. Atomic nuclei were treated as quantum particles interacting via a tight-binding-type potential. At $300 \mathrm{~K}$, the difference $\Delta a$ between lattice parameters of $3 \mathrm{C}-\mathrm{SiC}$ crystals with ${ }^{12} \mathrm{C}$ and ${ }^{13} \mathrm{C}$ amounts to $2.1 \times 10^{-4} \AA$. The effect due to $\mathrm{Si}$ isotopes is smaller, and amounts to $3.5 \times 10^{-5} \AA$ when replacing ${ }^{28} \mathrm{Si}$ by ${ }^{29} \mathrm{Si}$. Results of the PIMD simulations are interpreted in terms of a quasiharmonic approximation for the lattice vibrations.
\end{abstract}

PACS numbers: 71.20.Nr, 71.15.Pd, 63.20.Ry, 63.20.Dj

It is well known that the lattice parameters of two chemically identical crystals with different isotopic composition are not equal, lighter isotopes giving rise to larger lattice parameters. This is caused by a combination of two factors: the dependence of atomic vibrational amplitudes upon atomic mass, and the anharmonicity of the vibrations. The isotope effect on the lattice parameter is most important at low temperatures, due to the change of zero-point vibrational amplitude with atomic mass, and disappears in the high-temperature (classical) limit at $T>\Theta_{D}\left(\Theta_{D}\right.$, Debye temperature), where vibrational amplitudes are independent of the mass. In recent years, it has become feasible to measure the isotopic effect in lattice parameters of crystals with high precision. $\underline{\underline{1}}$ Most of the work has been performed on elemental crystals, although binary and multinary materials offer the attractive possibility of isotopic substitution on different atoms. $\stackrel{2}{=}$ Of these materials, $\mathrm{SiC}$, with 3 stable isotopes of $\mathrm{Si}$ and 2 of $\mathrm{C}$, plus about 70 different polytypes, is a paragon. Here, however, we confine ourselves to the simplest polytype: zincblende-like 3C-SiC.

$\mathrm{SiC}$ has been suggested for a number of applications exploiting many of its superlative properties, close to those of diamond $\stackrel{3.4}{=}$ Some of these applications take advantage of its hardness, large thermal conductivity, and low thermal expansion. Isotopically modified $\mathrm{SiC}$ may find applications exploiting the higher thermal conductivity ${ }^{\underline{5}}$ and the dependence of its hardness on isotopic composition. $\underline{6}$

Anharmonic effects in the vibrational properties of 3C$\mathrm{SiC}$ have been studied earlier in detail, e.g., the pressure and temperature dependence of phonon frequencies 7.8 and lifetimes $\frac{9,10}{10}$ The thermal expansion of cubic $\mathrm{SiC}$ (another anharmonic effect) has been studied in detail both experimentally ${ }^{11}$ and theoretically ${ }^{12,13}$, the latter using a quasiharmonic approximation (QHA) for the lattice vibrations.

Isotopic effects on the lattice parameters of crystals have been usually calculated by employing the
QHA and perturbative methods based on ab-initio techniques. ${ }^{2,14,15}$ An alternative to perturbational approaches in solids is the combination of the path integral formulation (to deal with the quantum nature of the nuclei) with electronic structure methods. The path integral approach to statistical mechanics allows one to study finite-temperature properties of quantum manybody problems in a nonperturbative scheme, even in the presence of large anharmonicities $\underline{16}$ An advantage of its combination with electronic structure methods is that both electrons and atomic nuclei are treated quantum mechanically in the framework of the Born-Oppenheimer approximation. $\stackrel{17,18,19}{1}$ The path-integral molecular dynamics (PIMD) method is based on an isomorphism between the quantum system under consideration and a classical one, obtained by replacing each quantum particle by a cyclic chain of classical particles, connected by harmonic springs, $\underline{16,20,21}$

When calculating properties of crystals with isotopically mixed composition, it is usually assumed that each atomic nuclei in the solid has a mass equal to the average mass. This kind of virtual-crystal approximation has been used in density-functional calculations, as well as in atomistic simulations based on path integrals, $, 2,21,22,23,24$ In fact, in earlier path integral simulations of diamond it was found that the results obtained by using this approximation are indistinguishable from those derived from simulations in which actual isotopic mixtures were considered. ${ }^{24}$

Here we extend earlier path integral calculations of the lattice parameter of group-IV solids ${ }^{21,23,24}$ (diamond, $\mathrm{Si}, \mathrm{Ge})$ to a IV-IV compound such as $3 \mathrm{C}-\mathrm{SiC}$. The electronic structure has been treated with an efficient tight-binding Hamiltonian, based on density-functional calculations ${ }^{25,26}$ Simulations were performed on a $2 \times$ $2 \times 2$ supercell of the $3 \mathrm{C}$-SiC face-centered cubic cell with periodic boundary conditions, including 64 atoms. For a given temperature and isotopic composition, a typical 


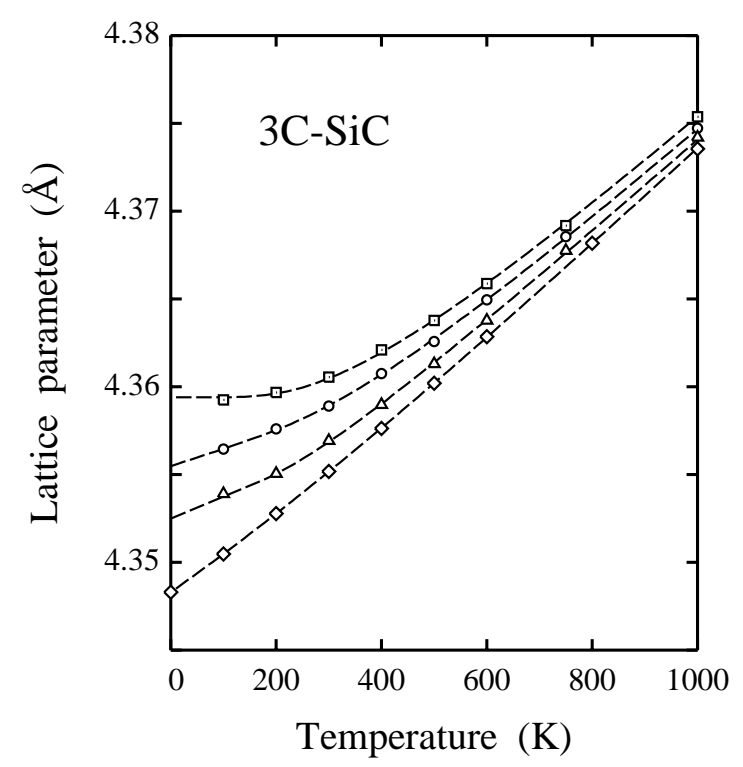

FIG. 1: Temperature dependence of the lattice parameter of $3 \mathrm{C}-\mathrm{SiC}$, as obtained from (a) PIMD simulations (squares), (b) simulations performed by setting the Si nuclei as classical particles (circles), (c) simulations carried out by considering classical C nuclei (triangles), and (d) all nuclei are assumed to be classical (diamonds). Lines represent empirical fits. The statistical error of the simulation results is less than the symbol size.

run consisted of $2 \times 10^{4}$ PIMD steps for system equilibration, followed by $4 \times 10^{6}$ steps for the calculation of ensemble average properties. Details on the actual implementation of the PIMD method to study structural and electronic properties of $\mathrm{SiC}$ were given elsewhere $\underline{26}^{26}$

First of all, we quantify the influence of quantum effects on the lattice parameter of cubic SiC. With this purpose we have performed simulations in which the atomic nuclei of either $\mathrm{C}$ or $\mathrm{Si}$ (or both) were considered as classical particles. We note that in the formalism used here, the classical limit for a given atomic nucleus is obtained by taking its mass as $M \rightarrow \infty$ (in fact, we took masses in the order of $10^{5} \mathrm{amu}$ ).

In Fig.1 we present the results of our molecular dynamics simulations for the various cases considered. First, we compare the results of the full PIMD simulations (quantum atomic nuclei, open squares) with those of classical simulations (diamonds). The zero-point motion induces an increase in the lattice parameter of $0.011 \AA$, which means a relative change of $2.5 \times 10^{-3}$. Second, we are interested in the lattice parameter obtained when nuclei of one of the elements ( $\mathrm{Si}$ or $\mathrm{C}$ ) are considered as classical and the other as quantum particles. Thus, triangles represent results for quantum $\mathrm{Si}$ and classical $\mathrm{C}$, whereas circles indicate data for quantum $\mathrm{C}$ and classical Si. By comparing these results with those found in the full PIMD simulations, we find for $T \rightarrow 0$ that the lattice parameter $a$ increases by $3.9 \times 10^{-3} \AA$ and $6.8 \times 10^{-3} \AA$, due to having considered $\mathrm{Si}$ or $\mathrm{C}$ as quantum particles, respectively (assuming in each case that atomic nuclei

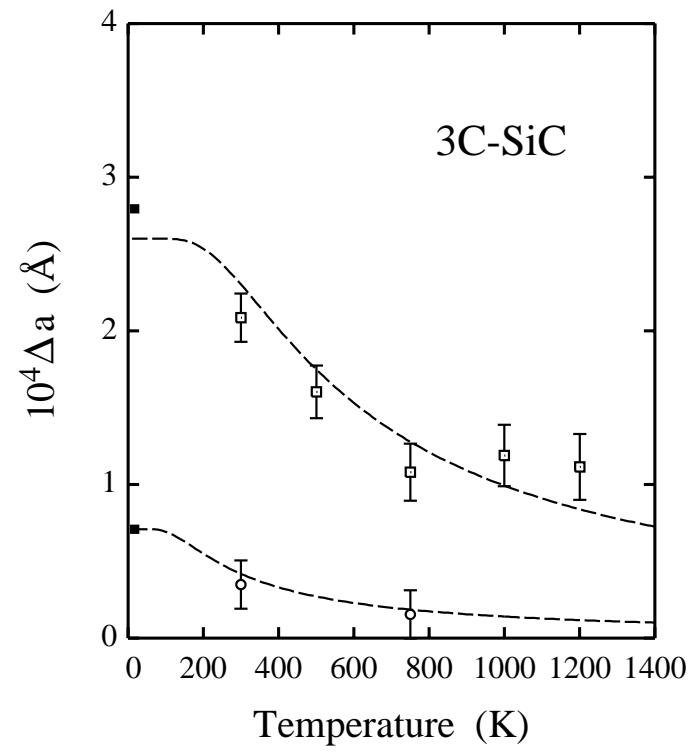

FIG. 2: Temperature dependence of the isotope effect on the lattice parameter of $3 \mathrm{C}-\mathrm{SiC}$. Squares represent the difference between lattice parameter of ${ }^{\text {nat }} \mathrm{Si}^{12} \mathrm{C}$ and ${ }^{\text {nat }} \mathrm{Si}^{13} \mathrm{C}$, whereas circles indicate the difference $\Delta a$ between ${ }^{28} \mathrm{Si}^{\text {nat }} \mathrm{C}$ and ${ }^{29} \mathrm{Si}^{\text {nat }} \mathrm{C}$. Filled symbols indicate the low-temperature values derived from Eq. (5). Lines represent fits of the simulation results to Eq. (1).

of the other element are quantum particles). We note that these results are similar to those presented earlier in Ref. 26, but here the simulations extended over runs 10 times longer in order to improve the accuracy in the lattice parameter, and the present error bars are smaller by a factor of $\sim 3$.

Results presented until now were obtained for $\mathrm{SiC}$ crystals with natural isotopic composition. We now turn to simulations of $\mathrm{SiC}$ crystals, in which one of the elements is taken to be isotopically pure. Thus, we carried out PIMD simulations of ${ }^{\text {nat }} \mathrm{Si}^{12} \mathrm{C}$ and ${ }^{\text {nat }} \mathrm{Si}^{13} \mathrm{C}$ (where the superscript "nat" refers to the mass of the natural isotopic composition) at several temperatures. Results for the difference $\Delta a$ between lattice parameters of both kinds of crystals are shown in Fig. 2 (squares). At room temperature, we find $\Delta a=2.1 \times 10^{-4} \AA$, which translates into a relative change $\Delta a / a=4.8 \times 10^{-5}$. Something similar has been done for ${ }^{28} \mathrm{Si}^{\text {nat }} \mathrm{C}$ and ${ }^{29} \mathrm{Si}^{\text {nat }} \mathrm{C}$ crystals, and the difference $\Delta a$ yielded by our simulations is much lower: at $300 \mathrm{~K}$ we found $\Delta a=3.5 \times 10^{-5}$ $\AA$, or $\Delta a / a=8 \times 10^{-6}$. The temperature dependence of $\Delta a$ can be fitted to a function with the shape of the mass derivative of a Bose-Einstein function, namely:

$$
\Delta a=C\left[1+\frac{2}{\mathrm{e}^{x}-1}\left(1-\frac{x \mathrm{e}^{x}}{\mathrm{e}^{x}-1}\right)\right]
$$

where $x=T_{a} / T$, and $C$ and $T_{a}$ are fit parameters. Dashed lines in Fig. 2 represent this function with $T_{a}=$ $1200 \mathrm{~K}$ (upper curve) and $600 \mathrm{~K}$ (lower curve). Eq. (1) is basically equivalent to Eq. (3) of Ref. 26 except that 
the adjustable parameter $b$ has been replaced by welldefined physical variables more convenient for the subsequent treatment. The minor deviations of the PIMD points from the fitted curves are not surprising since we have used a single Einstein oscillator fit. They could be decreased by using two oscillators ${ }^{27}$

The results of our PIMD simulations can be further analyzed in terms of a QHA for the lattice vibrations. In such an approximation, the lattice parameter $a(T)$ for a given isotopic composition at temperature $T$ can be derived by minimizing the Helmholtz free energy with respect to the crystal volume $\stackrel{2,22}{=}$ One finds

$$
a(T)=a_{\infty}+\frac{1}{3 B a_{\infty}^{2}} \sum_{n, \mathbf{q}} \gamma_{n}(\mathbf{q}) E_{n}(\mathbf{q}, T)
$$

where

$$
E_{n}(\mathbf{q}, T)=\frac{1}{2} \hbar \omega_{n}(\mathbf{q}) \operatorname{coth}\left(\frac{\hbar \omega_{n}(\mathbf{q})}{2 k_{B} T}\right)
$$

Here, $\omega_{n}(\mathbf{q})$ are the frequencies of the $n$th mode in the crystal, $B$ is the bulk modulus, $a_{\infty}$ is the zero-temperature lattice parameter in the limit of infinite atomic mass (classical limit), and $\gamma_{n}(\mathbf{q})=$ $-\partial \ln \omega_{n}(\mathbf{q}) / \partial \ln V$ is the Grüneisen parameter of mode $n, \mathbf{q}$. Then, at $T=0$ the difference $a(0)-a_{\infty}$ is given by

$$
a(0)-a_{\infty}=\frac{1}{6 B a_{\infty}^{2}} \sum_{n, \mathbf{q}} \hbar \omega_{n}(\mathbf{q}) \gamma_{n}(\mathbf{q})
$$

Let us consider now for simplicity two isotopically-pure monatomic crystals with a mass difference $\Delta M$. The difference between the corresponding lattice parameters, $\Delta a$, can be related to the zero-point renormalization, $a_{\text {nat }}(0)-a_{\infty}$, for the natural crystal. This can be achieved through a first-order expansion for the lattice parameter as a function of the mass $M$, and taking into account that the frequencies $\omega_{n}(\mathbf{q})$ scale as $1 / \sqrt{M}$. One finds for the change in lattice parameter at $T=0$ :

$$
\Delta a=-\frac{1}{2}\left(a_{\mathrm{nat}}(0)-a_{\infty}\right) \frac{\Delta M}{M_{\mathrm{nat}}} .
$$

This means that the low-temperature changes in $a$ due to isotopic mass can be obtained from the zero-point renormalization of the lattice parameter in the natural crystal.

For binary compounds such as $\mathrm{SiC}$, one can use a formula similar to Eq. (5) to obtain the separate contributions of each kind of atoms (i.e., Si or C). To first order, the contributions of both types of atoms will be additive. Then, from the difference $a_{\text {nat }}(0)-a_{\infty}$ discussed above, and presented in Fig. 1, we obtain in the low-temperature limit, using Eq. (5),$\Delta a=2.8 \times 10^{-4} \AA$ when replacing ${ }^{12} \mathrm{C}$ by ${ }^{13} \mathrm{C}$, and $\Delta a=7.0 \times 10^{-5} \AA$ for substitution of ${ }^{28} \mathrm{Si}$ by ${ }^{29} \mathrm{Si}$. These are the values shown in Fig. 2 as filled squares at $\mathrm{T}=0$.

We note that the virtual-crystal approximation has been employed in our simulations, i.e., for crystals with

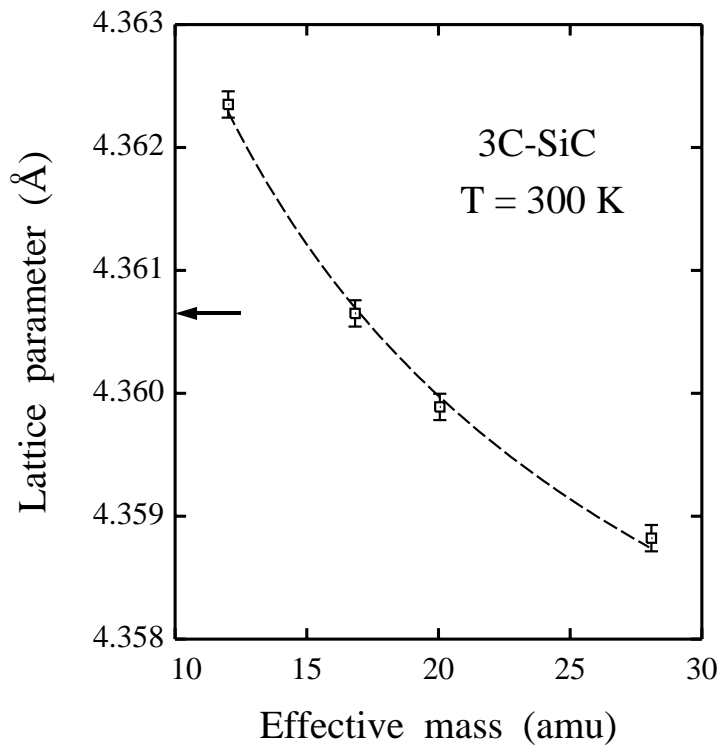

FIG. 3: Lattice parameter of $3 \mathrm{C}-\mathrm{SiC}$ as a function of $M_{\text {eff }}$ in a virtual-crystal approximation, in which all atoms in the simulation cell ( $\mathrm{Si}$ and $\mathrm{C}$ ) are assumed to have the same effective mass. Open squares indicate results of PIMD simulations in this approximation. The dashed line is a fit to Eq. (9). An arrow indicates the lattice parameter derived from PIMD simulations with $\mathrm{C}$ and $\mathrm{Si}$ atoms having their actual mean isotopic masses.

the natural isotopic composition of $\mathrm{Si}$ or $\mathrm{C}$ we have assumed that the atoms have the average mass. In this respect it is worthwhile considering the validity of assuming an effective mass $M_{\text {eff }}$ for all the atoms in a given crystal, to describe changes in the lattice parameter. In view of Eq. (4), one has: ${ }^{22}$

$$
\frac{a(0)-a_{\infty}}{a_{\infty}} \sim \frac{\hbar}{B V_{c}}\left\langle\omega_{n}(\mathbf{q})\right\rangle\left\langle\gamma_{n}(\mathbf{q})\right\rangle
$$

where $V_{c}$ is the volume of the primitive cell, and $\langle\ldots\rangle$ indicates an average over all branches of the Brillouin zone. Now we may assume a dependence of the average frequency on effective mass as

$$
\left\langle\omega_{n}(\mathbf{q})\right\rangle \sim M_{\mathrm{eff}}^{-1 / 2},
$$

which, in fact, is expected when one considers $M_{\text {eff }}$ as

$$
\frac{1}{M_{\mathrm{eff}}}=\frac{1}{2}\left(\frac{1}{M_{\mathrm{C}}}+\frac{1}{M_{\mathrm{S} i}}\right) .
$$

To check this point we have carried out PIMD simulations for $\mathrm{SiC}$ crystals with various effective masses for both $\mathrm{Si}$ and $\mathrm{C}$. The results are displayed in Fig. 3 as open symbols. For comparison with the result derived for $M_{\text {eff }}$ given by Eq. (8) $(\sim 16.8 \mathrm{amu})$, we also show those obtained by assuming an effective mass given either by the average $\left(M_{\mathrm{C}}+M_{\mathrm{S} i}\right) / 2$, or by the mass of $\mathrm{Si}$ or C. All these results can be fitted well to the expression

$$
a=b+\frac{c}{\sqrt{M_{\mathrm{eff}}}},
$$


where $b$ and $c$ are fit parameters. Such an expression can be expected from Eq. (4) when one considers an effective mass for both types of atoms, at temperatures $T \ll \Theta_{D}$ (for $\mathrm{SiC}, \Theta_{D} \sim 1100 \mathrm{~K}$ ). The actual lattice parameter of $\mathrm{SiC}$ yielded by the simulations above is indicated in Fig. 3 by an arrow. It coincides within error bars with that derived assuming $M_{\text {eff }}=16.8 \mathrm{amu}$, as given in Eq. (8). Note that taking the effective mass as the average of the masses (at about $20 \mathrm{amu}$ ) yields a lattice parameter clearly lower than the actual one obtained using the separate masses of $\mathrm{C}$ and $\mathrm{Si}$.

The agreement between the result for $M_{\text {eff }}=16.8 \mathrm{amu}$ and the real crystal can be interpreted in terms of perturbation theory as follows. Looking at Eq. (4), changes in the lattice parameter are mainly due to TO phonons, as can be derived from Fig. 9 in Ref. 13 for the appropriate values of $\gamma_{n}$ and Fig. 2 for the density of phonon states. The TO band in $\mathrm{SiC}$ is rather symmetric, 13 and according to second-order perturbation theory, ${ }^{22,28}$ mass fluctuations cause an increase in the high frequencies and a reduction of the low ones by a similar amount. All together the effect in the lattice parameter is expected to be negligible, as observed in the results of the simulations. If some effect appears due to mass fluctuations, it has to be of third or higher order, and should be less than the statistical uncertainty of our results. This gives further support to the virtual-crystal approximation for calculating lattice parameters of this kind of semiconductors.

In summary, we have calculated the isotope effect on the lattice parameter of cubic SiC by PIMD simulations. This procedure gives a quantitative estimation of such effect, which amounts to $\Delta a / a=4.8 \times 10^{-5}$ for replacement of ${ }^{12} \mathrm{C}$ by ${ }^{13} \mathrm{C}$, and to $\Delta a / a=8 \times 10^{-6}$ for substitution of ${ }^{29} \mathrm{Si}$ for ${ }^{28} \mathrm{Si}$. These results have been interpreted in terms of a quasi-harmonic approach for the lattice vibrations. The virtual-crystal approximation is found to be valid in all cases considered here. Second order perturbation theory, as implied by Eq. (5), has also been shown to be valid.

\section{Acknowledgments}

This work was supported by Ministerio de Ciencia e Innovación (Spain) through Grant No. FIS200612117-C04-03 and by CAM through project S$0505 / \mathrm{ESP} / 000237$.
1 A. Kazimirov, J. Zegenhagen, and M. Cardona, Science 282, 930 (1998).

2 A. Debernardi and M. Cardona, Phys. Rev. B 54, 11305 (1996).

3 W. J. Choyke, H. Matsunami, and G. Pensel, Silicon Carbide: Recent Major Advances (Springer, Heidelberg, 2003).

4 E. S. Saddow and A. Agarwal, Advances in Silicon Carbide Processing and Applications (Artech House, Norwood, MA, 2005).

${ }^{5}$ L. E. Berman, J. B. Hastings, D. P. Siddons, M. Koike, V. Stojanoff, and M. Hart, Nucl. Instrum. Methods Phys. Res. A 329, 555 (1993).

6 A. K. Ramdas, S. Rodriguez, M. Grimsditch, T. R. Anthony, and W. F. Banholzer, Phys. Rev. Lett. 71, 189 (1993).

7 D. Olego, M. Cardona, and P. Vogl, Phys. Rev. B 25, 3878 (1982).

8 D. Olego and M. Cardona, Phys. Rev. B 25, 3889 (1982).

9 A. Debernardi, C. Ulrich, M. Cardona, and K. Syassen, phys. stat. sol (b) 223, 213 (2001).

10 K. Karch, F. Bechstedt, P. Pavone, and D. Strauch, J. Phys.: Condens. Matter 8, 2945 (1996).

11 G. A. Slack and S. F. Bartram, J. Appl. Phys 46, 89 (1975).

12 D. N. Talwar and J. C. Sherbondy, Appl. Phys. Lett. 67, 3301 (1995).

13 K. Karch, P. Pavone, W. Windl, O. Schütt, and D. Strauch, Phys. Rev. B 50, 17054 (1994).
14 P. Pavone and S. Baroni, Solid State Commun. 90, 295 (1994).

15 S. Biernacki and M. Scheffler, J. Phys.: Condens. Matter 6, 4879 (1994).

16 D. M. Ceperley, Rev. Mod. Phys. 67, 279 (1995).

17 M. E. Tuckerman, D. Marx, M. L. Klein, and M. Parrinello, Science 275, 817 (1997).

18 R. Ramírez, E. Hernández, J. Schulte, and M. C. Böhm, Chem. Phys. Lett. 291, 44 (1998).

19 B. Chen, I. Ivanov, M. L. Klein, and M. Parrinello, Phys. Rev. Lett. 91, 215503 (2003).

20 M. J. Gillan, Phil. Mag. A 58, 257 (1988).

21 J. C. Noya, C. P. Herrero, and R. Ramírez, Phys. Rev. B 53, 9869 (1996).

22 M. Cardona and M. L. W. Thewalt, Rev. Mod. Phys. 77, 1173 (2005).

23 C. P. Herrero, Solid State Commun. 110, 243 (1999).

24 C. P. Herrero, J. Phys.: Condens. Matter 13, 5127 (2001).

25 D. Porezag, T. Frauenheim, T. Köhler, G. Seifert, and R. Kaschner, Phys. Rev. B 51, 12947 (1995).

26 R. Ramírez, C. P. Herrero, E. R. Hernández, and M. Cardona, Phys. Rev. B 77, 045210 (2008).

27 J. Manjón et al., Eur. Phys. J. B 40, 453 (2004).

28 F. Widulle, J. Serrano, and M. Cardona, Phys. Rev. B 65, 075206 (2002). 